Gesnerus 55 (1998) 17-32

\title{
Die Drehmaschinen in der Geschichte der Psychiatrie
}

Christian Müller

\section{Summary}

The history of the rotatory machine shows a distinct transformation during its short-lived use in psychiatric therapy from about 1806 to 1836 . Initially a variety of hypotheses were offered to explain its mode of action. Gradually the use of the method became a plain disciplinary measure. The technical features of the machine also changed. The adherents of both psychic and somatic treatment in psychiatry did not object to the method. Obviously, as a result of Conolly's no-restraint the use of the machine fell into disrepute. The scant case histories suggest that the machine was mainly used as a means of intimidation and deterrent. It is worth to be remembered that humane physicians in the middle of the 20th century still used deterring methods such as the aversion treatment of alcoholics.

\section{Zusammenfassung}

Die Geschichte der Drehmaschinen von ihrem Ursprung bis zu ihrem Verschwinden aus dem therapeutischen Arsenal (ca. 1806-1836) zeigt eine deutliche Wandlung. Während ursprünglich noch unklare Hypothesen zur Wirkungsweise vorlagen und auch die Technik der Maschine sich wandelte, wurde das Ganze schliesslich zu einem reinen Zuchtmittel. Es ist auffällig, dass alle «Schulen», d. h. sowohl die «Psychiker» wie die «Somatiker», nichts gegen diese Methode einzuwenden hatten. Dass die Drehmaschinen dann im Rahmen der internationalen Diskussion für und gegen das Conollysche No-restraint in Verruf gerieten, ist nicht verwunderlich. Aus den spärlichen Kasuistiken ist herauszulesen, dass es vor allem um Einschüchterung und

Prof. Christian Müller, Herrengasse 23, 3011 Bern 
Abschreckung ging, wobei nicht zu übersehen ist, dass abschreckende Methoden noch durch sehr humane Ärzte Mitte des 20. Jahrhunderts angewendet wurden, z. B. die Apomorphin-Ekelbehandlung bei Alkoholismus.

\section{Einleitung}

In den meisten Übersichtsarbeiten und Lehrbüchern zur Geschichte der Psychiatrie finden sich Angaben, dass in den ersten zwei Jahrzehnten des letzten Jahrhunderts Drehmaschinen in der Behandlung von Geisteskranken zur Anwendung kamen. Ihre Erwähnung wird meistens mit den Adjektiven «barbarische Methode» versehen (E. Kraepelin ${ }^{1}$, F. G. Alexander und S. C. Selesnik ${ }^{2}$, E. H. Ackerknecht ${ }^{3}$, S. Kornfeld ${ }^{4}$, M. Schrenk ${ }^{5}$ ). Tatsächlich ergreift den Leser ein Schaudern, wenn er in den einschlägigen Artikeln um 1810 nicht nur eine Beschreibung, sondern auch künstlerisch angefertigte Zeichnungen dieser Maschinen zu Gesicht bekommt (s. Abb. 1).

Folgen wir vorerst einmal einer Beschreibung, welche E. Horn ${ }^{6} 1818$ verfasst hat:

«Die Drehmaschine (das Drehbett) ist seit zehn Jahren in der mit dem königlichen CharitéKrankenhaus verbundenen Irrenanstalt unter die Zahl der Heilmittel aufgenommen, und seit dieser Zeit bei mehreren Hundert Geisteskranken und bei manchem mit vielem Nutzen in Gebrauch gezogen worden.»

«Die Wirkung dieser Drehmaschine ist zusammengesetzt. Der auf derselben so befestigte Kranke, dass er darin aufrecht sitzt oder horizontal liegt, wird in schnellen Schwingungen um seine Achse gedreht, wobei der Kopf den peripherischen, die Füsse den Zentralkreis umschreiben. Ein gesundes Individuum, welches den Wirkungen dieser Maschine sich aussetzt, kann nicht länger wie einige Minuten das höchst unangenehme Gefühl ertragen, welches durch diese eigentümliche Bewegung hervorgebracht wird.»

Wie schnell drehte sich diese Maschine? Darüber gibt ein Artikel von F. $\operatorname{Müller}^{7}(1823)$ Auskunft. Er schreibt:

«Die Drehmaschine ... habe ich mit viel Mühe und beträchtlichem Kostenaufwand für das Juliusspital erhalten. Sie ist von grossen Meistern der Mechanik verfertigt und macht in einer Minute vierzig Umschwingungen in einem Kreis von vierzig Fuss und hat keinen

1 Kraepelin E.: Hundert Jahre Psychiatrie. Springer-Verlag, Berlin 1918.

2 Alexander F. G. und Selesnik S. C.: Geschichte der Psychiatrie. Verlag Diana, Zürich 1969.

3 Ackerknecht E. H.: Kurze Geschichte der Psychiatrie. Verlag E. Enke, Stuttgart 1985.

4 Kornfeld S.: Geschichte der Psychiatrie. In: Handbuch der Geschichte der Medizin, Bd. 3, hg. von Neuburger u. Pagel, Jena 1905.

5 Schrenk M.: Über den Umgang mit Geisteskranken. Springer-Verlag, Berlin/Heidelberg 1973.

6 Horn E.: Öffentliche Rechenschaft über meine zwölfjährige Dienstführung als zweiter Arzt des königlichen Charité-Krankenhauses zu Berlin. Berlin, Realschulbuchhandlung 1818.

7 Müller F.: Bemerkungen über die bisher berühmtesten empirischen Mittel in psychischen Krankheiten. Zeitschr. f. die Anthropologie, erstes Vierteljahrheft, 97-207, Leipzig, 1823. 


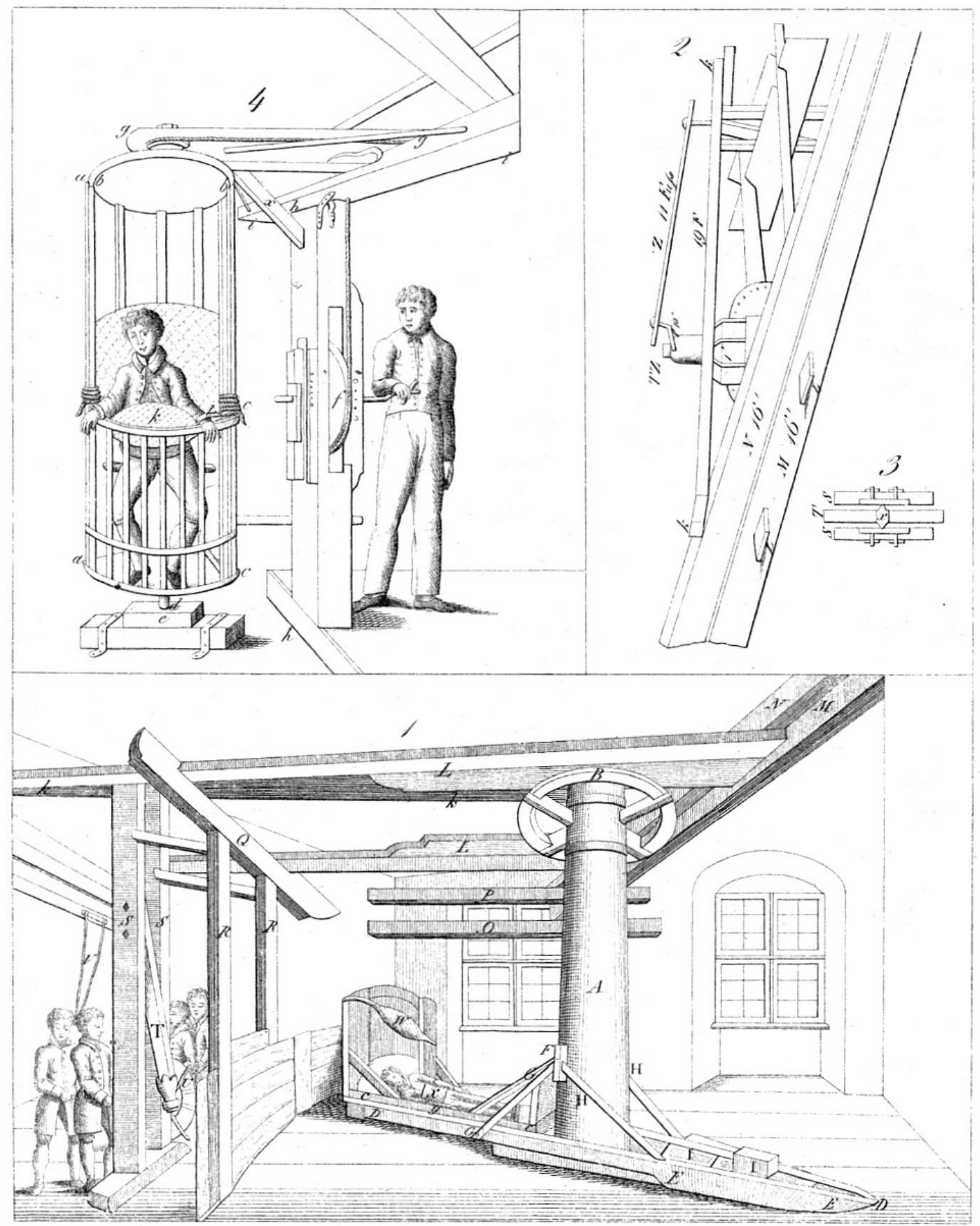

Abb. 1. Aus: Schneider P. J., Entwurf zu einer Heilmittellehre gegen psychische Krankheiten, Tübingen 1824, Taf. I. 
Fehler als nur den, dass fünf bis sechs Personen nothwendig sind, ihr schnelle Bewegung zu geben.»

Offenbar gab es aber nicht nur einen Typus von Drehmaschinen, sondern manche Spitalpsychiater modifizierten die Anleitungen, so wenn R. Hayner ${ }^{8}$ 1818 schreibt:

«Ich weiss, dass man Maschinen der Art hat, die sich weit geschwinder bewegen lassen als die meine. Dafür kosten aber auch jene viel Geld, sind sehr künstlich und nehmen einen grossen Platz ein. Die meinige kostet etwa 5 bis 6 Taler, ist ganz einfach, kann in jedem Stübchen angebracht werden, lässt sich ... so schnell drehen, dass ein homo sanae mentis in fünf Minuten den ganzen Magen erbricht ...»

Geht man die psychiatrische Literatur aus den Jahren 1810 bis 1840 durch, so gewahrt man, dass die Drehmaschinen regelmässig, wenn auch oft nur kursorisch als Therapeutikum erwähnt werden. Meist berufen sich die Autoren auf den englischen Psychiater M. J. Cox ${ }^{9}$, von dem noch die Rede sein soll. Häufig wird schlicht von den eigenen Konstruktionen berichtet, ohne Hinweis auf den Ursprung dieser Maschine. Nur C. F. W. Roller ${ }^{10}$ wird ausführlicher.

In neueren psychiatriegeschichtlichen Arbeiten geht J. Starobinski ${ }^{11}$ auf unser Thema ein; auch bei G. Windholz ${ }^{12}$ und bei F. J. M. Schmid ${ }^{13}$ finden wir Angaben, während G. Wunderlich ${ }^{14}$ erstaunlicherweise die Drehmaschine kaum erwähnt. Je mehr man sich mit dem Thema der Drehmaschinen beschäftigt, um so grösser wird der Wunsch, Näheres über ihre Entstehung und ihre Anwendung in der Psychiatrie zu erfahren und sich nicht mit der Ablehnung als barbarische Methode zu begnügen. Es stellen sich nämlich verschiedene Fragen:

1. Woher stammte die Idee, wer hat sie entwickelt resp. von andern übernommen?

2. Welches waren die theoretischen Überlegungen, die zur Anwendung führten?

8 Hayner R.: Über einige mechanische Vorrichtungen, welche in Irrenanstalten mit Nutzen gebraucht werden können. Zeitschr. f. psychische Ärzte, drittes Vierteljahrheft, 339-366, 1818.

9 Cox M. J.: Praktische Bemerkungen über Geisteszerrüttung. Aus dem Englischen übersetzt. Renger'sche Buchhandlung, Halle 1811.

10 Roller C.F. W.: Die Irrenanstalt nach allen ihren Beziehungen. Verl. Chr. F. Müller, Karlsruhe 1831.

11 Starobinski J.: Dokumenta Geigy Acta psychosomatica Nr. 4, 74-79, 1966

12 Windholz G.: Psychiatric Treatment and the Condition of the Mentally Disturbed at Berlin's Charité in the Early Decades of the Nineteenth Century. In: History of Psychiatry 6,157-176, 1995.

13 Schmidt F. J.M.: Die Entwicklung der Irrenpflege in den Niederlanden. Verl. MurkenAltrogge, Herzogenrath 1985.

14 Wunderlich G.: Krankheits- und Therapiekonzepte am Anfang der deutschen Psychiatrie. Abh. zur Geschichte der Medizin und der Naturwissenschaften. Matthiesen Verlag, Berlin, Heft 41,1981. 
3. Welches war die Verbreitung in Europa?

4. Während welcher Zeitspanne wurden diese Drehmaschinen überhaupt verwendet?

5. Gibt es Indikationskriterien und kasuistische Darstellungen von Behandlungen mit der Drehmaschine?

\section{Ursprung der Drehmaschine}

H. J. Laehr ${ }^{15}$ erwähnt, dass man bei Celsus ${ }^{16}$ bereits den Rat finde, Geisteskranke durch Schaukeln zu beruhigen. Und auch bei Avicenna soll es Hinweise darüber geben, dass schwingende Bewegungen für den Geisteskranken wohltätig sein könnten. Öfters wird in der alten Literatur auf eine Arbeit von C. G. Kratzenstein hingewiesen $(1775)^{17}$. Nicht zuletzt soll der berühmte Boerhaave ${ }^{18}$ nicht nur das Untertauchen in Wasser, sondern auch das Drehen des Kranken empfohlen haben.

Konkretere Angaben zum Ursprung finden sich dann aber in dem bekannten Buch von R. Hunter und J. Macalpine ${ }^{19}$. Wir erfahren dort, dass als einer der ersten J. C. Smyth (1741-1821) 1787 in einer Arbeit «An Account of the Effects of Swinging employed as a Remedy in the Pulmonary Consumption» von einer Anwendung bei Geisteskranken gesprochen hat.

E. Darwin (1731-1802), der Grossvater von Charles Darwin, hat dann diese Idee aufgenommen und in seinem Werk, das 1796 erschien, der Schwingmethode das Wort geredet.

Schliesslich war es J. M. Cox (1763-1818) ${ }^{20}$, der in seinem erfolgreichen Buch von 1806 eine ausführliche Beschreibung dessen gibt, was er «the

15 Laehr H.J.: Über Irresein und Irrenanstalten. Halle, C.E.M. Pferer 1852.

16 Frau Prof. J. C. Wilmans verdanke ich den Hinweis, dass im 18. Kapitel des dritten Buches «De medicina», wo Celsus eine Form von Insania der Phrenitis gleichstellt, der Satz auftauche: «Confert etiam aliquid ad somnum silanus juxta cadens vel gestatio post cibum et noctu maximeque lecti suspensi motus.» (Auch ist ein Springbrunnen, der in der Nähe plätschert, dem Schlaf etwas förderlich oder auch das Getragen- (resp. Gefahren)werden nach der Mahlzeit und in der Nacht, und am besten helfen leicht schwingende Bewegungen eines schwebenden Bettes.).

17 Kratzenstein C. G.: Novum medicinae genus nimirum vim centrifugam ad morbos sanandos applicatam more geometrarum proponit. Kopenhagen 1765.

18 Dies schreiben z. B. Alexander und Selesnik. Allerdings habe ich bei Boerhaave in dem Kapitel «Von der Raserei» Paragraph 1123 nur gefunden, dass er das Stürzen ins Meer und das Untertauchen empfiehlt, das so lange fortgesetzt werden solle, als der Kranke es ertragen könne (Boerhaave H.: Kurze Lehrsätze über Erkennung und Heilung der Krankheiten. Aus dem Lateinischen übersetzt. Henning'sche Buchhandlung, Gotha u. Erfurt 1831).

19 Hunter R. und Macalpine J.: Three Hundred Years of Psychiatry. Oxford University Press, London/New York/Toronto 1963.

20 Loc. cit. (9). 
rotatory machine» nennt. Psychiater, die später diese Methode anwendeten, vor allem in Deutschland, aber auch in Skandinavien, beriefen sich jeweils auf Cox, dessen Buch in verschiedene Sprachen übersetzt wurde.

An diesem Punkt angelangt, muss aber auf eine Schwierigkeit der Übersetzung hingewiesen werden. Während wir bei J. C. Smyth, E. Darwin und J. M. Cox immer nur von «swinging chair» hören, was in den deutschen Übersetzungen von Cox dann mit «Schaukel» übersetzt wird, handelt es sich bei den eingangs erwähnten Beschreibungen von E. Horn und anderen nicht um ein Schaukeln, sondern um ein Um-eine-Achse-Drehen. Ob allen späteren Autoren, die sich bei der Beschreibung ihrer Drehmaschinen auf Cox beriefen, bewusst war, dass dieser resp. seine Vorgänger nicht unbedingt nur das Drehen, sondern auch das Schaukeln gemeint haben, muss offen bleiben. Dass ein Schaukeln im Sinne von Wiegen einen beruhigenden Einfluss auf erregte Kranke haben kann, liegt auf der Hand, während für die Rotation auf dem Stuhl oder auf dem Bett bis zu Übelkeit und Bewusstlosigkeit ganz andere Vorgänge ins Auge zu fassen sind. Dieselben Maschinen haben übrigens in den verschiedenen Ländern verschiedene Bezeichnungen erhalten. So spricht B. Rush ${ }^{21}$ von «gyrator», und in Frankreich redet man von «chaise giratoire».

Wir sehen also, dass das Schaukeln von Geisteskranken eine alte Tradition hat, das Drehbett und der Drehstuhl aber erst zu Beginn des 19. Jahrhunderts, vor allem in Deutschland, aufgetaucht sind.

Fast möchte man bedauern, dass die Idee des sanften Schaukelns oder Wiegens nicht mehr Aufmerksamkeit gefunden hat. Man hätte ja ohne weiteres an die Wirksamkeit einer «legitimen Regression» denken können; wenn nämlich der erregte Geisteskranke wie ein Säugling gehegt und gewiegt wird. Ist es denkbar, dass ein derartiges Vorgehen wieder aufgegriffen wird? Im Rahmen der «körperzentrierten Therapien» wohl nicht so unsinnig.

\section{Theoretische Überlegungen}

Dass es sich bei dem Wiegen des Kranken um eine Wiederholung der mütterlichen Zuwendung handeln könnte, würde heute jeder Psychiater annehmen. Diesen Gedanken habe ich indessen in der älteren Literatur nirgends gefunden. Wohl aber werden immer wieder verschiedene psychologische

21 Rush B.: Medical Inquiries and Observations upon the Diseases of the Mind. 4. Aufl., Philadelphia Gregg 1830. 
Überlegungen gemacht. So schreibt z.B. M.J. Cox ${ }^{22}$ in seinem grundlegenden Buch:

«Nach wenigen Umdrehungen sah ich die Beruhigung des Körpers und der Seele und einen sanften Schlaf erfolgen; oft zeigte sich zuerst einiger Schwindel, nach welchem dann ein sehr erfrischender Schlummer eintrat, ein Erfolg, welcher bei verrückten Kranken immer sehr wünschenswert ist und der sonst nur mit der grössten Schwierigkeit hervorgebracht wird.»

Bei E. Horn ${ }^{23}$ finden wir nun ganz deutlich die Auffassung, dass es um eine Erweckung von unangenehmen Empfindungen gehe:

«Nützlich wird diese Wirkung bei der Behandlung der Geisteskranken durch Erwecken einer widrigen Empfindung, durch ein lebhaftes und ungewohntes Einwirken auf das Gemeingefühl, durch die Erweckung der Furcht, die teils der Anblick des ganzen Apparates und seiner Gebrauchsart, teils die Besorgnis, herausgeschleudert und gefährlich verletzt zu werden, hervorruft durch Erweckung des Schwindels, der Übelkeit und des Erbrechens. Durch diese Wirkungen tritt die Drehmaschine in die Reihe der indirekten psychischen Heilmittel. Je grösser die Empfänglichkeit des Kranken ist, je weniger er schon an Mittel dieser Art sich gewöhnte, je lebhafter der ganze Apparat ihm imponierte, je unangenehmer und lästiger ihm seine Anwendung ist, um desto wohltätiger zeigen sich die Wirkungen dieses Heilmittels.»

A. Haindorf $\mathrm{f}^{24}$ macht andere theoretische Überlegungen. Er rückt die Bedeutung des Schwindels in einen psychologischen Zusammenhang. So schreibt er:

«Die Aktion des Schwindels auf das grosse Gehirn oder den inneren Sinn ist aber jedesmal total und erregt mit aller Energie das Organ zur heftigsten Reaktion. Schwindelerregende Mittel müssen deshalb bei Hirnaffektionen von der grössten Wirksamkeit sein, zumal wenn zugleich durch Brech- und Purgiermittel auch das gastrische System mitrevoltiert wird. Es kann deshalb nicht leicht eine so Totalergreifung des Organismus geben, als wenn Schwindel und das gastrische System revoltierende Mittel, zweckmässig miteinander verbunden, zugleich in Anwendung gebracht werden; indem letztere vermöge des grossen Konsens des gastrischen Systems mit dem Gehirn mächtig auf dieses zurückwirken und folglich dieses mitrevoltieren.»

Bei andern Autoren wiederum finden wir die Vorstellung, dass das Hirn durch das Schwingen zusammengedrückt und die Blutversorgung somit verändert werde. Für einige soll es vor allem um die Evakuierung von Mageninhalt gehen, was für die psychische Störung von Nutzen sein könne. Zum Erbrechen äussert sich auch P. J. Schneider $(1824)^{25}$, wenn er schreibt:

«Vorzüglich gute Dienste leistet aber die Schaukel nicht nur in solchen Fällen, wo unfolgsame Kranke durchaus alle Arzneien verweigern, sondern auch da, wo sie solche wirklich nicht nehmen können, wenn sie z.B. in einem bewusstlosen Zustande sind oder aus andern Ursachen nicht zu schlingen vermögen; denn oft hängt das Leben davon ab, dass schnell

22 Loc. cit. (9).

23 Loc. cit. (6)

24 Haindorf A.: Versuch einer Pathologie und Therapie der Geistes- und Gemüthskrankheiten. b/Gottl. Braun, Heidelberg 1811.

25 Schneider P. J.: Entwurf zu einer Heilmittellehre gegen psychische Krankheiten. b/H. Laupp, Tübingen 1824. 
Erbrechen erregt werde; auch die Empfindlichkeit des Magens befindet sich für die gewöhnlichen Vomitive in einem der Paralyse analogen Zustande, und hier vermag jene herrliche Bereicherung unseres akalogischen Apparats wirklich wunderbare Hilfe zu leisten.»

\section{Bei J. M. Cox lesen wir übrigens noch:}

«Da in einigen Fällen von Verrücktheit die Seelenkrankheit von der Tätigkeit des Herzens abzuhängen oder sich nach derselben zu richten scheint, sodass der vernünftige Zustand zurückkehrt, wenn die Zahl der Pulsschläge unter das Normal sinkt, und Verrücktheit eintritt, wenn sie über dasselbe steigt, hat sich die Schaukel vermöge ihres Einflusses auf den Blutumlauf hier als ein äusserst wohltätiges Mittel gezeigt.»

\section{Und weiter:}

«Da man das Erbrechen lange Zeit unter die vorzüglichsten Mittel in der Verrücktheit gerechnet hat, würde die Schaukel, brächte sie auch nur diese Veränderung im Körper hervor, schon von sehr grossem Werte sein.»

Die bisher erwähnten theoretischen Überlegungen werden bei J. Hirsch ${ }^{26}$ noch etwas abgewandelt. Er schreibt in seinem Artikel, dass man bei den Müllern besonders häufig Geisteskrankheiten feststelle, und er führt es darauf zurück, dass die Ursache ein Schwindel sei, der sich «bei der Betrachtung der unablässig sich gleichbleibenden einförmigen Bewegung des Räderwerks bilde». Der Schwindel beruhe auf der in Unordnung gebrachten Tätigkeit einiger Reizungen der Muskeln, z.B. der des Magens, durch Berauschung oder durch Brechmittel. Er meint schliesslich, dass sogar die «Drüsen des Gehirns» selber Schwindel erregen können, indem ihre Bewegungen mit dem grossen Zirkel von Reizungsbewegungen verkettet sind. Die Seekrankheit gibt einen überzeugenden Beweis der Affektion der Nervengeflechte in der Präkordialgegend durch deren in Unordnung gebrachte Tätigkeit vermöge der Reizungen, deren einförmige Bewegung das Bedingende derselben sei.

J. Hirsch spricht von einer Affektion des Nervengeflechts durch einförmige oder gleichmässige Schwingungen und behauptet dann, die überreizten Schwingungen der Nerven könnten dadurch beruhigt werden, dass künstlich erregte Gegenschwingungen erfolgen. J. Hirsch erweist sich also darin als ein Anhänger der antagonistischen Therapiemethoden.

1940 finden wir bei K. Bonnhoeffer ${ }^{27}$, einem der späten Nachfolger von E. Horn in Berlin, eine Rechtfertigung der Drehmaschinen. Er weist darauf

26 Hirsch J.: Beschreibung eines neuen Heilapparates für Geisteskranke in der Irrenheilanstalt zu St. Georgen bei Baireut. In: Zeitschr. f. die Anthropologie, erstes Vierteljahrheft, 456-459, Leipzig 1823.

27 Bonnhoeffer K.: Die Geschichte der Psychiatrie in der Charité im 19. Jahrhundert. In:Z.ges. Neurol. u. Psychiat., 168: 43-51, 1940. 
hin, dass durchaus humane Ärzte wie J. C. Reil oder E. Horn sich zum Teil phantastische theoretische Vorstellungen machten über die gewaltsamen Einwirkungen auf Gemeingefühl, Haut, Auge und Ohr, was bei Horn zur Erfindung der Drehmaschine geführt habe. Und weiter:

\begin{abstract}
«Diese Theorie von der therapeutischen Wirkung eindrucksvoller Sinneserregungen auf Geisteskrankheiten sieht fast nach einem physiologisch-psychologischen Versuch aus, überkommene, mittelalterliche Zwangs- und Strafmethoden in Einklang zu bringen mit der sich allmählich durchsetzenden ärztlichen und humanen Betrachtungsweise, die aber noch nicht imstande war, sich therapeutisch den erregten Geisteskrankheiten gegenüber durchzusetzen. Es ist neben den Erfahrungen über die tatsächlichen Erfolge der heroischen Verfahren des Drehstuhls und der Eiswasserbegiessungen offenbar auch die Hilflosigkeit gegenüber den unruhigen Kranken, gesteigert durch unzulängliche Unterbringungsverhältnisse, durch zahlenmässig und qualitativ unzulängliches Pflegepersonal, was die klärenden Zwangsmassnahmen bei einer im Grunde humanen Grundeinstellung aufrecht erhielt.»
\end{abstract}

Für uns ist es interessant festzustellen, dass Bonnhoeffer in einer Fussnote bemerkt:

«Da man wohl annehmen darf, dass die Psychiater jener Zeit in der Beurteilung von eingetretenen Remissionen nicht weniger urteilsfähig waren als wir Heutigen, wird man an der tatsächlichen Wirkung dieser Gewaltkuren nicht zu zweifeln brauchen. Wahrscheinlich handelt es sich um ähnliche Schockeinwirkungen, wie sie auf medikamentösem Wege mit Insulin und Cardiazol erreicht werden.»

J. Starobinski ${ }^{11}$ erwähnt noch einen andern theoretischen Ansatz, indem er J. C. Heinroth zitiert, der meint, die Drehmaschine sei ein Aufrüttelungsmittel ersten Ranges und könne eine geschwundene Rezeptivität wiederherstellen, sie sei indiziert, sobald der Kranke ganz in sich versinke und sich vom Geist der Schwere übermannen lasse.

Zusammengefasst ergibt sich also, dass vor allem in Deutschland zu Beginn des 19. Jahrhunderts das Erregen von Unlust und Angst als therapeutisches Agens der Drehmaschinen betrachtet wurde, dass aber auch andere physiologische Wirkungen erwogen wurden, so z.B. die Heilsamkeit des Schwindels, das Erbrechen von «schädlichem» Mageninhalt, die Veränderung der Blutzufuhr zum Gehirn.

\title{
3. Verbreitung der Drehmaschinen in den europäischen psychiatrischen Spitälern um 1820
}

Zur Abklärung dieser Frage wurde die Literatur nach Angaben über das Vorhanden- oder Nichtvorhandensein solcher Apparate in den verschiedenen Institutionen abgesucht. 1811 beispielsweise finden wir die Bemerkung, wonach es in Sonnenstein bei Pyrna einen «Maschinensaal mit mechanischen 
Heilmitteln» gegeben habe ${ }^{28}$. Es klingt sehr wahrscheinlich, dass dort auch eine Drehmaschine vorhanden war. Laut D. Jetter ${ }^{29}$ soll es um 1820 in den Anstalten Siegburg, Hochheim, Heina, Winnental solche Drehmaschinen gegeben haben. Über Heina hat im einzelnen H. Siefert berichtet ${ }^{30}$. Hingegen finden wir bei J. H. F. Autenrieth $(1807)^{31}$ bei seiner Beschreibung der Zwangsmittel, die er in der Klinik in Tübingen angewendet hat, keine Erwähnung von Drehmaschinen. Ausführlich beschreibt er die Einrichtung des Palisadenzimmers und der Autenriethschen Birne, die eine traurige Berühmtheit unter den Zwangsmitteln erleben sollte. Aber offenbar hat er die Drehmaschine nicht gekannt oder nicht verwenden wollen. Dass diese in England verbreitet waren, erstaunt nicht, wenn man bedenkt, dass einer ihrer Erfinder, M. J. Cox, dort gelebt hat. Bei G. M. Burrows ${ }^{32}$ finden wir 1828 die Bemerkung:

«The rotatory machine is met with now in most British public asylums. But its results are very differently reported, some speaking most favourably of it, some dubiously, and with others it has fallen into disuse. Without wishing to extoll ist merits more than it is due, this diversity of opinion cannot be the fault of the remedy. It is reasonable to suppose that the same degree of attention in selecting the cases to try it on and the rules prescribed for applying it have not been equally observed in different institutions, and hence these contradictions.»

Dass die Drehmaschinen auch in Skandinavien bekannt und gebraucht waren, erfährt man aus einem Artikel von A. M. Söderström ${ }^{33}$. Er beschreibt, wie ein schwedischer Chefarzt, Engstroem, 1829 die Direktion eines psychiatrischen Spitals, Vadsdena, übernimmt und sofort auch den Plan fasst, eine Drehmaschine anzuschaffen. So schreibt dieser 1829:

\begin{abstract}
«Wenn man an der Spitze eines grossen Spitals ist, muss man alle Instrumente zur Verfügung haben, welche dazu dienen können, die Geisteskranken zu heilen. Es bedarf auch mehrerer Ärzte, welche eine gute Ausbildung haben und die Behandlungsformen kennen, beispielsweise dasjenige der «svängunga», d. h. eines Drehstuhls. Ich verlange deshalb von der Direktion, einen solchen Stuhl anzuschaffen, und wenn wir auch nicht genau dessen Konstruktion kennen, so können wir doch uns am Exemplar eines andern schwedischen Spitals, Danvik, orientieren.»
\end{abstract}

Dass in Frankreich die Drehmaschinen kaum zur Verwendung kamen, muss betont werden. Wir finden denn auch nur ganz spärliche Mitteilungen

28 Nach Thom H.: Zur Geschichte der Psychiatrie im 19. Jahrhundert. Berlin 1983.

29 Persönliche Mitteilung von Prof. D. Jetter, Medizinhist. Institut Köln.

30 Siefert H.: Kloster und Hospital Haina. Hessisches Ärzteblatt, Jg. 32, Heft 11, 1971.

31 Autenrieth J.H. F.: Versuche für die praktische Heilkunde aus den clinischen Anstalten von Tübingen. Cotta'sche Buchhandlung, Tübingen 1807.

32 G. M. Burrows: Commentaries on Insanity. p. 599-605, Underwood, London 1828.

33 Söderström A. M.: Svängstolen vid Vastena Hospital Hygiea. Medicinsk Tidskrift, Bd. 98: 545-567, 1936. Die Übersetzung dieses Artikels besorgte Dr. D. Söderström in Nant-surCorsier, dem ich hiermit meinen Dank abstatte. 
in den zeitgenössischen Arbeiten. So lesen wir bei E. Esquirol ${ }^{34} 1827$ lakonisch:

«Ein Wort zur Maschine von Darwin. ... Cox hat sie empfohlen, Hufeland und Horn benützen sie in Berlin. In Genf soll ein Apparat existieren, welcher Odier die Gelegenheit gab, die Wirkung zu beobachten. Ein Dr. Martin aus Lyon hat mir gesagt, er sei entsetzt gewesen über die Unfälle, welche Kranke erlebten, wenn sie in eine solche Maschine gespannt wurden. Sie wurden ohnmächtig.»

$\mathrm{Zu}$ dieser kritischen Bemerkung passt auch die Tatsache, dass bei der Lektüre des «Journal inédit» des Marquis de Sade ${ }^{35}$ über seinen Aufenthalt 1807-1808 und dann wieder ab 1814 im psychiatrischen Spital Charenton bei Paris: ... keine Spur eines Drehbetts entdeckt werden kann, wohl aber die kalte Dusche. Collins bitterböse Kritik an den Zwangsmassnahmen in Charenton bezieht sich nicht auf die Drehmaschine.

Dass in Belgien der Drehstuhl bekannt war, geht aus der Abhandlung «Über die Phrenopathie» von J. Guislain ${ }^{36}$ hervor, die er 1828 publizierte. Er erwähnt dann die Vorgänger, d.h. E. Darwin und M. J. Cox, und schreibt:

«Der Drehstuhl, dessen wir uns bedienen, ist eine andere Form dessen von Cox. Er besteht aus einem Lehnstuhl von festem Holz, der sich auf einem Zapfen dreht.»

So muss also angenommen werden, dass in Belgien im Jahre 1828 der Drehstuhl verwendet wurde. Eigenartig erscheint im übrigen, dass bei einer zweiten Ausgabe seines Lehrbuches $1880^{37}$ der Drehstuhl zwar immer noch erwähnt wird, aber dass der Autor dort deutlich ausspricht, «seit langem habe ich ihn nicht mehr verwendet».

Wie aber steht es mit der Schweiz? E. Esquirol erwähnt Odier, der diese Maschine in Genf verwendet habe. In einem Bericht von C. Otto ${ }^{38}$ über die psychiatrischen Einrichtungen in der Schweiz zwischen 1820 und 1823 finden wir die interessante Mitteilung, dass er in Zürich nicht nur die Zwangsjacke, sondern auch eine Drehmaschine gesehen habe. Die bei-

34 Esquirol E.: Des maladies mentales. Volume 1. Arno Press, New York, Times Company New York 1976.

35 De Sade: Journal inédit. Collection folios Gallimard. Ed. 1970. Der Marquis de Sade berichtet in diesem Tagebuch von seinem Aufenthalt in dem psychiatrischen Spital Charenton von 1807 bis 1808 und dann wieder um 1814. Es findet sich trotz aller Kritik an den Einrichtungen von Charenton kein Hinweis auf die Existenz einer Drehmaschine. In der im selben Bändchen publizierten «Notice sur l'établissement de Charenton» von Collins, geschrieben 1812, wird zwar ebenfalls über barbarische Methoden geklagt, erwähnt werden aber nur die Dusche aus grosser Höhe, die Peitsche, das bain permanent, aber keine Drehmaschine.

36 Guislain J.: Abhandlung über die Phrenopathien. Aus dem Französischen von Dr. Wunderlich. J.F. Rieger u. Co., Stuttgart/Leipzig 1838.

37 Guislain J.: Leçons orales sur les phrénopathies. $2^{\mathrm{e}}$ édition, tome second, Gand/Paris, Baillière et fils, 1880.

38 Otto C.: Reise durch die Schweiz, Italien, Frankreich, Grossbritannien und Holland. A. Campe, Hamburg 1825. 
den Quellen aus Genf und Zürich sind die einzigen zur Schweizer Psychiatrie gefundenen.

Was schliesslich Österreich betrifft, so konnte nur in einem Artikel von D. Jetter ${ }^{39}$ die Erwähnung gefunden werden, dass er auf Architekturzeichnungen aus dem Jahre 1820 in Wien die Bemerkung gelesen habe, dass dort ein geräumiges Zimmer für «mechanische Vorrichtung» vorgesehen gewesen sei.

Dass in bezug auf die Verwendung der Drehmaschinen in Europa auch eindeutig negative Meinungen in der Zeitspanne zwischen 1810 und 1840 auftauchen, zeigen verschiedene Texte. J. M. Leupold ${ }^{40}$ berichtet z.B. 1825 von seinen Einwänden und meint, dass «man über kurz oder lang der Überzeugung werde, wie wenig diese mechanischen Zwangsmittel anwendbar sind ...».

Weit geht L. Buzzorini ${ }^{41} 1832$ in seiner Ablehnung, wenn er schreibt:

«... Zwangsbeinkleider, Drehstuhl, Drehbett, hohles Rad usw., welche zum Teil ein so fürchterliches Ansehen haben, so gewaltsam in ihren Wirkungen sind, und durch ihren Zweck und ihre Einwirkung an jene barbarische Behandlung der Irren unserer Vorfahren erinnern, die man vedrängt zu haben sich rühmt, aber unbefangen betrachtet kann man in ihnen nichts anderes als (etwa zeitgemässe) Surrogate für Ketten und Peitsche, und als Heilmittel in ihrer Anwendung nur eine medicinisch verfeinerte Prügelkur erblicken.»

Aber auch Eschenburg ${ }^{42}$ in Lübeck äussert sich 1832 sehr kritisch. Um so erstaunlicher ist es, dass der anerkannte Reformator der nordamerikanischen Psychiatrie B. Rush ${ }^{43}$ die Drehmaschine noch 1835 empfiehlt.

\section{Die Dauer der Verwendung der Drehmaschine}

Die Anfänge reichen mit E. Darwin in die letzten Jahre des 18. Jahrhunderts. Als sicheres Datum können wir mit Cox das Jahr 1806 bestimmen. Im Anschluss daran ist es wohl zu einer Verbreitung in Europa gekommen, wobei es nicht unzulässig ist, die Jahre zwischen 1810 und 1830 als die Hauptperiode der Anwendung des «Gyrators» festzulegen. Nach 1830 werden die Mitteilungen seltener, ja es tauchen nun bereits Bemerkungen auf, dass die Drehmaschinen ausser Gebrauch geraten seien.

39 Jetter D.: Wiener Irrenhausprojekte. Fortschr. Neurol. Psychiatrie, 49: 43-92, 1981.

40 Leupold J .M.: Über Leben und Wirken und über psychiatrische Klinik Nürnberg b. Riegel und Wiessner, 1825.

41 Buzzorini L.: Grundzüge einer Pathologie und Therapie der psychischen Krankheiten. J. G. Cotta, Stuttgart/Tübingen 1832.

42 Ich entnehme diese Angabe dem aufschlussreichen Band von K. H. Reger und H. Dilling: Psychiatrie in Lübeck: das 19. Jahrhundert. Verl. Schmidt-Römhild. Lübeck 1984.

43 Loc. cit. (21). 
Der berühmte C. G. Carus ${ }^{44}$, Freund von Goethe, kommt in seinen Lebenserinnerungen auf einen Besuch in der Charité in Berlin zu sprechen, und es erstaunt, dass er bereits im Jahr 1817 das Verschwinden der Drehmaschinen registriert:

«Soviel ist wenigstens gewiss, dass mit Horn dieses System der Behandlung dort aufgehört hat und auch in keiner anderen Anstalt mit dieser Konsequenz wieder durchgeführt worden ist ...»

Wahrscheinlich hat zu diesem von Carus bemerkten Verschwinden des Drehstuhls aus der Charité in Berlin die Tatsache beigetragen, dass Horn, der Chef der Psychiatrie in dieser Klinik, nach einem Todesfall in einen Prozess verstrickt wurde. Allerdings ging es nicht um einen Unfall bei der Anwendung der Drehmaschine, sondern eine junge Frau erstickte in dem Hornschen «Sack». Dieser Prozess löste grosse Kontroversen aus, und der junge Schopenhauer, der damals Geisteskranke in der Charité beobachtete, wurde offenbar durch diesen Todesfall beeindruck ${ }^{45}$.

Bei M. Viszanik ${ }^{46}$ endlich finden wir konkrete Angaben über das Verschwinden der Drehmaschine aus dem therapeutischen Arsenal. Er berichtet nämlich, dass er 1845 in den psychiatrischen Spitälern Prags keinen Drehstuhl gesehen habe und dass er auch in den deutschen Anstalten Sonnenstein, Sachsenberg-Mecklenburg, ja auch in Marsberg keine Drehmaschine mehr gefunden habe. Erstaunlich ist es, dass offenbar Schweden das Land war, wo diese Therapie am längsten fortgeführt wurde.

Zusammengefasst kann man sagen, dass diese «Schocktherapie» (K. Bonnhoeffer) rund 30 Jahre lang angewandt wurde. Damit reiht sie sich punkto Dauer in die Reihe der modernen Schockbehandlungen ein. Man kann grosso modo sagen, dass die Sakelsche Insulinschockbehandlung von 1936 bis 1966 eine Blüte erlebte, dann aber völlig aufgegeben wurde ${ }^{47}$. Für Klaesis Schlafkur ${ }^{47}$ wiederum gilt der 30-Jahre-Zeitraum, hat sie doch Anwendung gefunden in den Jahren 1922 bis ca. 1954, bis zu jenem Zeitpunkt nämlich, da sie durch die Neuroleptikatherapie verdrängt wurde.

44 Carus C. G.: Lebenserinnerungen und Denkwürdigkeiten. Verl. Brockhaus, Leipzig 1865.

45 Zentner M.: Die Flucht ins Vergessen: Die Anfänge der Psychoanalyse Freuds bei Schopenhauer. Darmstadt 1995. In diesem Buch finden sich ausgezeichnete und genau recherchierte Angaben zu dem berühmten Prozess gegen Horn und die Einflüsse der Beobachtung von Kranken auf den jungen Schopenhauer.

46 Viszanik M.: Die Irrenheil- und Pflegeanstalten Deutschlands, Frankreichs samt der Cretinenanstalt auf dem Abendberge in der Schweiz. C. Gerold, Wien 1845.

47 Siehe die Angaben dazu in Müller C.: De l'asile au centre psychosocial. Verl. Payot, Lausanne 1997. 


\section{Indikationen und Kasuistik zur Therapie mit Drehmaschinen}

Es ist nicht einfach, eine genaue Liste der Indikationen aus den alten Publikationen herauszukristallisieren. Bei E. Horn ${ }^{48}$ beispielsweise lesen wir, dass es sich um Anfälle der Tobsucht, aber auch der periodisch wiederkehrenden Manie gehandelt habe:

«Schwermütige, störrische und unfolgsame Kranke wurden dadurch zur Ordnung und Folgsamkeit gebracht, Wahnsinnige mit der Neigung zu Selbstmord wurden dadurch wohltätig erschüttert und umgestimmt.»

Schneider ${ }^{49}$ fügt hier noch die Epilepsie hinzu und schliesst den «Blödsinn» als durch die Drehmaschine unbehandelbar aus. Dass keine spezifischen Indikationen in der alten Literatur ersichtlich sind, verwundert kaum, wurden doch fast sämtliche Therapien, also auch zum Brechreiz führende, beruhigende, purgierende Mittel, Zur-Ader-lassen usw., wahllos bei allen «Seelengestörten» angewandt.

\section{Zur Kasuistik:}

Bereits bei M. J. Cox ${ }^{50}$ finden sich Fallbeschreibungen. So schildert er die Behandlung eines vierzigjährigen Mannes, den man heute wohl als bipolar psychotisch bezeichnen würde. Cox beschreibt, dass er immer niedergeschlagener wurde, nachdem er vorher ausschweifend und flatterhaft gewesen war. Er weigerte sich, Medikamente einzunehmen. «Tag und Nacht brachte er abwechselnd mit Sträuben gegen die angewendeten Zwangsmittel und mit heftigem Geschrei zu.» Cox schildert dann, dass es nur mit Hilfe von fünf Männern gelang, den Patienten in die Drehmaschine zu bringen.

«...A Als die Bewegung vermehrt wurde, veränderten sich seine Gesichtszüge, er wurde blass und der Urin ging ihm ab....

Nach ein paar schnellen Umdrehungen folgte Erbrechen, sein Kopf sank auf die Schulter und sein ganzer Körper schien der Kraft beraubt. Aus der Schaukel wurde er durch einen einzigen Aufwärter zu Bett gebracht, wo er sogleich in Schlaf verfiel. Nachdem er neun Stunden ohne Unterbrechung geschlafen hatte, erwachte er ruhig und erfrischt.... Er gehörte bald unter die Rekonvaleszenten, Gesundheit des Körpers und der Seele kehrten zurück ...»

Dr. W. Ruer ${ }^{51}$, der das Irrenhaus Marsberg leitete, berichtet 1819:

«Th. Buddenkott, eines Bauern Sohn aus der Gegend bei Münster gebürtig, sieben und zwanzig Jahre alt, wurde am dritten Oktober 1816 als Geisteskranker in die hiesige Irrenanstalt aufgenommen....

48 Loc. cit. (6).

49 Loc. cit. (29).

50 Loc. cit. (9).

51 Ruer W.: Nachrichten über die Irrenanstalt Marsberg. In: Zeitschr f. psych. Ärzte, 54-62, Leipzig 1819. 
1815 fiel das Signal zum Ausbruch seiner jetzigen Gemüthszerrüttung. In der ersten Zeit war er still, brütete düster vor sich hin; selten ward er gesprächig. Nachdem man sich von der Wirklichkeit seiner Geisteszerrüttung überzeugt, und ihn seinen Verwandten zurückgegeben hat, wechselt auch jetzt noch oft ein stilles, stummes, lässiges Betragen mit einem nur zu wilden wüsten Benehmen ab: dann treibt er sich rastlos umher, unternimmt bald diese, bald jene zwecklose Handlung, und wird ungestüm bis zur Unbändigkeit; durch ernste Weisungen, und nach Umständen durch heftige Drohungen, lässt er sich zwar noch wohl leiten, jedoch hält es schwer, ihn dauerhaft zu regelmässigen Arbeiten anzuhalten...

Bei Aufnahme des Kranken, dessen Übel nun bereits ein Jahr gedauert hatte, bemerkte ich hinsichtlich des psychischen und physischen Habitus noch Folgendes. ...

Der Kranke sprach murmelnd und gemüthlich vor sich hin; in seinen Handlungen verriet er keine Heftigkeit; mangelnder Ideenzusammenhang, mangelnde Aufmerksamkeit auf äussere Gegenstände, ein Insichgekehrtsein und die Zeichen mangelnder Selbstbestimmung sprachen sich unverkennbar in seinem ganzen Wesen aus. Er fragte und antwortete sich oft selbst....

Die ersten Tage hindurch wurde Patient in einer etwas abgesonderten Wohnung zur bessern Beobachtung allein gelassen. Er wurde meist kurz und laut angeredet. Ich liess ihn zur bestimmten Ordnung im Essen, Trinken, Aufstehen und Schlafen etc. anhalten, täglich zu gewissen Stunden unter steter Aufsicht ins Freie führen, um sich hier in körperlichen Arbeiten zu üben, wozu er durch ernstes Anreden und Drohungen gezwungen werden musste....

Als Arznei erhielt er vorläufig eine Auflösung von Brechweinstein, bis zur erregten Übelkeit. Bei verweigertem Gehorsam liess ich ihn mit Erfolg zu wiederholten Malen in den Drehstuhl führen. Er begehrte alsbald heraus, und versprach Folgsamkeit. So weit ging das ärztliche Verfahren bis zum 21sten Oktober, wo ich schon einen etwas heitern Blick und weniger Aufgetriebenheit des Gesichtes an ihm zu bemerken glaubte. Er gehorchte, ohne dass Drohung nöthig war, redete zuweilen etwas mehr in Zusammenhang, und es zeigten sich einige Spuren von Aufmerksamkeit....

In dieser Zeit bemerkte ich immer mehr auffallende Spuren herannahender Besserung. Das Aussehen des Kranken wurde heiterer; seine Rede zusammenhängender; das starre Vorsichhinblicken und Murmeln während der Arbeiten hatte nachgelassen, und das verkehrte Lesen unterliess der Kranke jetzt ganz.»

W. Ruer beschreibt dann den Fortgang der Besserung und berichtet, dass der Patient im Dezember 1817 geheilt entlassen werden konnte.

Offensichtlich war der Effekt der Drehmaschinenbehandlung ein rein abschreckender. Der Patient verhielt sich «vernünftiger» unter der Drohung der Wiederholung des qualvollen Experimentes. Derselbe Ruer beschreibt ein Jahr später in der gleichen Zeitschrift für psych. Ärzte von Nasse ${ }^{52}$ den Fall einer jungen Frau. Nach unseren heutigen Kriterien würden wir wahrscheinlich von einer Katatonie reden. Ruer beschreibt die Wirkung des Drehstuhls:

«Den elften und vierzehnten September liess ich sie in den Drehstuhl bringen: nach ungefähr zwanzig Kreis-Schwingungen und darauf erfolgtem plötzlichen Innehalten, rollte sie die Augen hin und her, war sehr ängstlich, aufmerksamer auf die äussere Umgebung und gelobte Folgsamkeit und Reinlichkeit an. Sie blieb den Tag über ruhiger; Nachmittags trat ungefähr eine halbe Stunde lang ein hellerer Zwischenraum ein. Den zwölften und dreizehnten September der vorige Zustand. Am vierzehnten nach wieder vorgenommenen

52 Ruer W.: Übersicht über die in der Irrenanstalt zu Marsberg im Jahr 1819 behandelten Kranken. In: Zeitschr. f. psych. Ärzte, 311-319, Leipzig 1820. 
Kreisbewegungen im Drehstuhl der nämliche Erfolg wie am eilften. Sie wurde strenger zu zweckmässigen Beschäftigungen, als Küchenarbeiten u.s.w., angehalten; auch wurde sie gezwungen, das Verunreinigte jedesmal wiederum selbst zu reinigen. In dieser Zeit hielt sie oft Stunden lang die linke Hand in dem Nacken fest, und so wie man dieselbe von da wegzog, führte sie sie schnell wieder dahin.»

1823 schreibt F. Müller aus Würzburg ${ }^{53}$ in der Nasse'schen Zeitung zur Anwendung der Drehmaschine:

«Ich habe zwar bis jetzt noch nicht oft genug Versuche damit gemacht, um ihr das Lob, was ihr Cox und Andere beilegen, gänzlich absprechen zu können; doch folgte auf ihren Gebrauch weder Schlag, noch Erbrechen. Länger als vier Minuten getraute ich mir die Operation nicht fortzusetzen, weil Blauwerden des Gesichts, Schwerathmen und Furcht des Erstickens eintraten, ich mochte die Kranken liegend oder sitzend in die Maschine bringen.»

Und schliesslich sei der schwedische Psychiater Engström erwähnt ${ }^{54}$. In seinem Journal von 1832 werden mehrere Fälle mit Anwendung der Drehmaschine erwähnt. Die Indikationen scheinen folgende gewesen zu sein: schwere Erregungszustände, katatoner Stupor, gelegentlich zur Disziplinierung unbotmässiger Kranker. Die Rotationszeit habe ca. 5-6 Minuten betragen. Beobachtet wurden weder Veränderung der Gesichtsfarbe noch des Pulses, wohl aber der ermüdende und vomitive Effekt. Allerdings wird die Geschwindigkeit der Drehung nicht erwähnt.

Ein Beispiel: Im Januar 1832 tritt eine Patientin ein, die als manisch bezeichnet wird. Sie sei aufsässig, zornwütig, sie zerreisse alles, sie schreie und rufe und wolle nicht gehorchen. Auf dem Drehstuhl ergibt sich vorerst keine Änderung. Nach 8 bis 10 Minuten wird sie ruhiger, schweigt, leidet an Schwindel, fühlt sich schwach, aber ohne Erbrechen. Sechs Monate später wird die Drehmaschine nochmals verwendet und provoziert Schwindel und Erbrechen. Sie wird dann ruhiger, legt sich nieder. Wie sie wieder schreit, bedroht man sie mit der Wiederholung der Drehmaschine.

Den «Erfolg» der Drehmaschinen-Behandlung im Vergleich mit anderen zeitgenössischen Heilverfahren zu messen ist unmöglich, da wir zu den damals üblichen Methoden (Sturzbad, Aderlass, Brechweinstein etc.) nur einzelne Beobachtungen haben. Als Psychiater, die wir heute über sehr potente Mittel verfügen, um hocherregte Kranke zu dämpfen, sollten wir den Stab nicht voreilig über unsere Vorgänger und ihre Drehmaschinen brechen. Sie waren sicher ehrlich bestrebt, dem entsetzlichen Ausser-sich-Sein des Psychotischen einen festen Riegel zu schieben.

53 Loc. cit. (7).

54 Siehe den Artikel von Söderström (33). 